\title{
Arachnid types in the Zoological Museum, Moscow State University. I. Opiliones (Arachnida)
}

\section{Типы паукообразных в Зоологическом музее МГУ. I. Opiliones (Arachnida)}

\author{
Kirill G. Mikhailov \\ К.Г. Михайлов \\ Zoological Museum MGU, Bolshaya Nikitskaya Str. 2, Moscow125009 Russia. E-mail: mikhailov2000@gmail.com \\ Зоологический музей МГУ, ул. Большая Никитская, 2, Москва 125009 Россия.
}

KEY WORDS: arachnids, harvestmen, museum collections, types, holotypes, paratypes.

КЛЮЧЕВЫЕ СЛОВА: паукообразные, сенокосцы, музейные коллекции, типы, голотипы, паратипы.

ABSTRACT: A list is provided of 19 holotypes and 92 paratypes belonging to 25 species of Opiliones. They represent 14 genera and 5 families (Ischyropsalidae, Nemastomatidae, Phalangiidae, Sabaconidae, Trogulidae) and are kept in the Zoological Museum of the Moscow State University. Other repositories housing the remaining types of the respective species are listed as well.

РЕЗЮМЕ: Представлен список 19 голотипов и 92 паратипов, относящихся к 25 видам сенокосцев (Opiliones). Они принадлежат к 14 родам и 5 семействам (Ischyropsalidae, Nemastomatidae, Phalangiidae, Sabaconidae, Trogulidae) и хранятся в Зоологическом музее МГУ. Также перечислены другие хранилища, в которых размещены другие типы указанных видов.

\section{Introduction}

The Zoological Museum of the Moscow State University was established in 1791 as a small Cabinet of Natural History. At present, 225 years later, the Museum consists of seven research departments which harbour ca 10,000,000 fixed animal specimens, including the types of ca 7,000 species. The Department of Invertebrates exists officially since 1931 as a place of storage of various invertebrate collections, insects excluded. The oldest specimens, shells and possibly corals, are kept here since the beginning of the $19^{\text {th }}$ century. Invertebrate type collections include holo-, paraand syntypes of more than 3,000 species, but published lists or catalogues are currently available only for oligochaete worms [Perel', Sokolskaya, 1972], mollusks [Ivanov, Sysoev, 2000], phoronids [Pakhnevich et al., 2013] and a few groups of crustaceans [Borutzky, 1972; Vinogradov, Vinogradov, 2006]; some of these publications need revision. Numerous arachnid and myria- pod types, as well as most of the crustacean types have never enjoyed published catalogues.

Traditionally, the following handwritten information sources are accepted in the Museum, at least so since the 1930's: (1) department acquisition book (Fig. 1), (2) numerous inventory books on diverse invertebrate groups (see Fig. 2 for Opiliones), and (3) type cards (Fig. 3). Regrettably, only a small part of this information has been digitalized.

This paper starts a series of lists/catalogues of arachnid types kept at the Museum. The arachnid collection considered was founded in the 1860's and presently contains more than 200,000 specimens of arachnids alone, Acari excluded [Mikhailov, 2016].

Contrary to spiders which in Moscow constitute the largest collection among the Russian and ex-USSR museums, the harvestman collection is moderate, being smaller than those of both the Zoological Institute, Russian Academy of Sciences in St. Petersburg and the Institute of Animal Systematics and Ecology, Siberian Branch, Russian Academy of Sciences in Novosibirsk. Experts in opilionid taxonomy have never worked in Moscow. So, hardly surprisingly, the type collection mostly represents donations of ex-USSR specialists like N.Yu. Snegovaya (Baku, Azerbaijan), A.N. Tchemeris (Tomsk, Russia), speleozoologist S.I. Ljovuschkin (Moscow, Russia), as well as specimens accessed from J. Martens (Mainz, Germany), the latter based on the comprehensive material obtained from Russia and other ex-USSR territories from zoologist S.I. Golovatch (Moscow, Russia), partly during their joint expeditions. In the Museum, the harvestman type collection (Fig. 4) is kept separately from the main collection of this group (Fig. 5).

The following indices are used for arachnids in the inventory books of the Zoological Museum, Moscow State University: Ta - spiders (Aranei, or Araneae), $\mathrm{Tb}$ - scorpions (Scorpiones), Tc - solpugids (Solifugae), Td — mites and ticks (Acari), Tk — harvestmen 


\begin{tabular}{|c|c|c|c|c|c|c|}
\hline \multirow[b]{2}{*}{110.} & \multirow{2}{*}{ 11.08.2015 Aiplyedr: Paradoxosomatidee, Sllanehere sp. } & \multicolumn{4}{|c|}{$\angle$} & \multirow[t]{2}{*}{141} \\
\hline & & 4 & anys. & ac.2. Toesbase & & \\
\hline 111 & 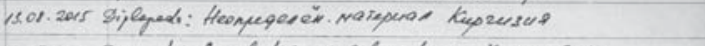 & 2eps & $-m$ & $\cdots-$ & 150,250 & \\
\hline 112 & 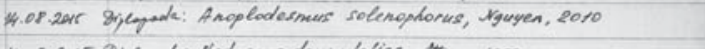 & 10, 3re, 4jü. & $\cdots$ & $\cdots$ & 150,12 & $\int 2007$ \\
\hline 1131 & 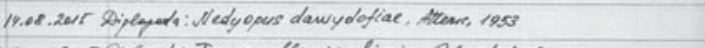 & 2ts, jear & $\ldots$ & $\cdots$ & 150,12 & Se708, 2x09 \\
\hline$M 4$ & 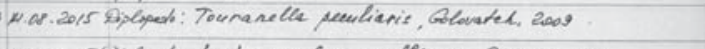 & 206,24 & - & - & 150,126 & Seno \\
\hline 115 & 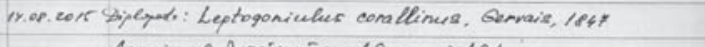 & 18,98 & $\cdots$ & $\cdots$ & 180,12 & S2z11 \\
\hline 116 & 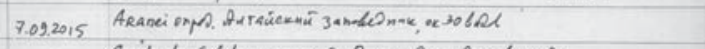 & $-100 \mathrm{z}$ & $-1 \cdot$ & 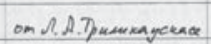 & 150.51 & $\begin{array}{l}\text { kapryothe } \\
\text { notumen }\end{array}$ \\
\hline 13. & 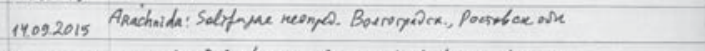 & $10^{\circ}$ & $-1-$ & Om.A.B. Domo mupele & 150.52 & kapraseamor \\
\hline 118 & 25.e9.201580pequed: Polydesmus lignavi, Lohmander, 1936 & 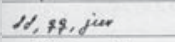 & $\cdots$ & or C.2. Toubara & $15,0,250$ & SeF18-2441 \\
\hline Ho & 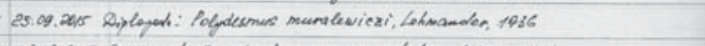 & $211,2 y, 4$ jice. & $\cdots$ & $-\cdots-$ & 150,12 & S2742-2744 \\
\hline 120 & 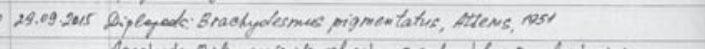 & & $\cdots$ & $\rightarrow-$ & 100,125 & $\int 2 \times 45-2755$ \\
\hline 11 & 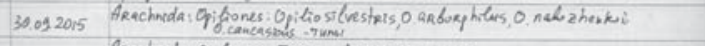 & $8 x y$. & -2 & om HW Cherebar- & 480.12 & Tk-47-54 \\
\hline 122. & 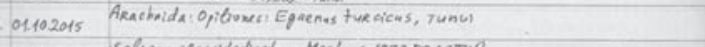 & $10+17$ & $-1-$ & om ham, Marycure & 1501251 & Tk. 55.56 \\
\hline 123. & 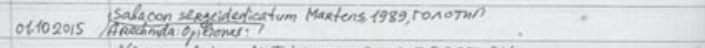 & 107 & $-1-$ & an JMaetens & 180.11 & $T k-57$ \\
\hline 121 & 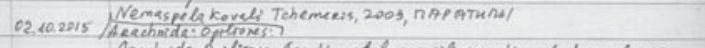 & 10111 & -5 & omA Herepruar & 1801251 & $T k-58.59$ \\
\hline 125. & 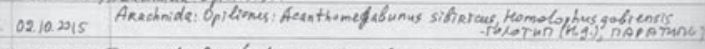 & 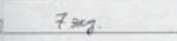 & $x$ & $-1-$ & 250.1251 & $T k 62.66$ \\
\hline 126 & 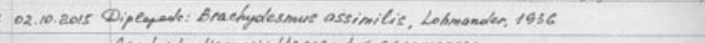 & $86,99 \cdot j u \mathrm{v}$ & $\cdots$ & or cus Thereare & 250,250 & $\rho 2756-2798$ \\
\hline 127 & 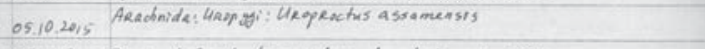 & 1 & 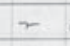 & om KBMakseba & 18.0251 & $T_{m}-1$ \\
\hline 128 & 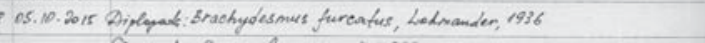 & di.99.juer. & $\cdots$ & or C. Z Therbase & 150,250 & $\int 2499-2832$ \\
\hline 129. & O610.2015 Cirripedia Bryozesina Rossi MAPATU17 & 1 (repmagnount) & $-1-$ & om CAKcoracela & 18.011 & $\mathrm{Ng}-1222$ \\
\hline 130 & 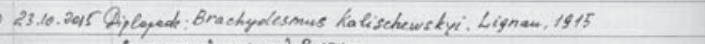 & \%.,99,jur & $\cdots$ & or C.4. Toubare & & S2833- \\
\hline 131. & 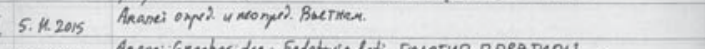 & $300 \%$ & $-1-$ & $\operatorname{m} 2.8 .4 \mathrm{gna} \alpha$ & $\begin{array}{l}\text { 75.14. } \\
45.0 .51 .\end{array}$ & - \\
\hline 132. & 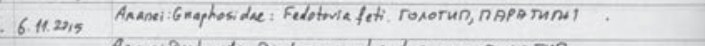 & 8 & + & 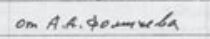 & 18.0 .11 & $T_{4}-7743.7745$ \\
\hline 133. & 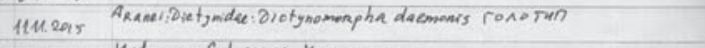 & 1 & 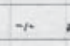 & om C. Ecotewry & 180.11 & $T_{4}-7746$ \\
\hline 134 & 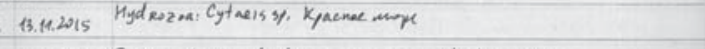 & 2 kownem & $\rightarrow$ & on B.M.WLANentiv & $-1-$ & EQ-169 \\
\hline 135. & 13.11.2015 Qiplopart: Brachydesmus superus, Lateel, 1884 & 511,1124 & $\cdots$ & or C.2.Joeobare & 180,2 & $\rho 3032,3033$ \\
\hline 136 & 6 16.11.2015 Dipbeade: Hyleoglomeris homplhmaiensis Paratypes & $-16,12$ & $\rightarrow-$ & - ar C.2.Touatra & 160.12 & $\rho 2712$ \\
\hline 137 & 7 16.11.2015 Riplopodt: Hyleoglomeris aurea & 11.19 & 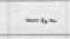 & $\cdots$ & 150,12 & $\int 27 / 3$ \\
\hline 138 & 8 16.11.2015 Siplequeds: Hyleoglomears cavicole. & $2 N, 49$ & $\cdots$ & $\cdots$ & 160,12 & S2HY \\
\hline 139 & 19.11-2015 Dipbyeds: Bnachydesmus kravadzei Panchype Won type & 18,11 & $\rightarrow$ & $-\cdots$ & 18. 0,2 & $\int 3035,3036$ \\
\hline 140 & 10 19.11.2015 Aplopeds: Brachyolesmeess simpler & 18 & $\rightarrow$ & $\rightarrow \cdot$ & is 0,125 & $\int 3037$ \\
\hline \multirow{2}{*}{\multicolumn{2}{|c|}{14119.112015 Qiplopede: Bractydesmus sinplus }} & $73 \%, 698$ & $\cdots$ & $\cdots$ & $\cdots$ & $53038-3040$ \\
\hline & & & & & & Te. Mry $4767-10 \mathrm{Na} \times 250 *$ \\
\hline
\end{tabular}

Fig. 1. An acquisition book of the ZMMU Invertebrate Department.

Рис. 1. Книга поступлений отдела беспозвоночных Зоологического музея МГУ.

(Opiliones), $\mathrm{Tl}$ — pseudoscorpions (Pseudoscorpiones), Tm - whip scorpions (Uropygi; currently no types).

Altogether, types of 25 species representing 14 genera and 5 families (Ischyropsalidae, Nemastomatidae, Phalangiidae, Sabaconidae, Trogulidae) are listed below, including 19 holotypes and 92 paratypes; 53 inventory numbers have been allotted. All opilionid species names are valid and currently available.

\section{Methods}

The following sequence of data is accepted in the type catalogue: species name in the original description; reference to the first description; list of types with labels; list of other collections where the remaining types of the respective species are kept; remarks; current taxonomic status of the species.

Label abbreviations are put in angled brackets, $<>$, when necessary. Author's comments to the labels are given in square brackets, []. An English translation of the original Russian labels is provided in figure brackets, \{\} .
Museum or personal collection data are abbreviated as follows: CJMM - work collection (= Arbeitssammlung) of Jochen Martens (Mainz, Germany); ISEA - Institute of Animal Systematics and Ecology, Siberian Branch, Russian Academy of Sciences (Novosibirsk, Russia); IZBA - Institute of Zoology, National Academy of Sciences (Baku, Azerbaijan); NHMW Naturhistorisches Museum Wien (Vienna, Austria); RCNS - reference collection of Nataly Snegovaya (Baku, Azebaijan); SMF — Senckenberg Museum (Frankfurt am Main, Germany); TNU - Department of Invertebrates, Taurida National University (Crimea, Russia); TTU-Z - Invertebrate Zoology, Museum of Texas Tech University (USA); ZIN — Zoological Institute, Russian Academy of Sciences (St. Petersburg, Russia); ZMMU — Zoological Museum, Moscow State University (Moscow, Russia). In the ZMMU, museum inventory numbers for Oplilones are indexed Tk put at the beginning of the museum labels. Labels are given literally, mistakes are indicated in comments.

The following translations of the administrative units are used herein: Area for "область, oblast", District for "район, rayon", Province for "край, kray". 


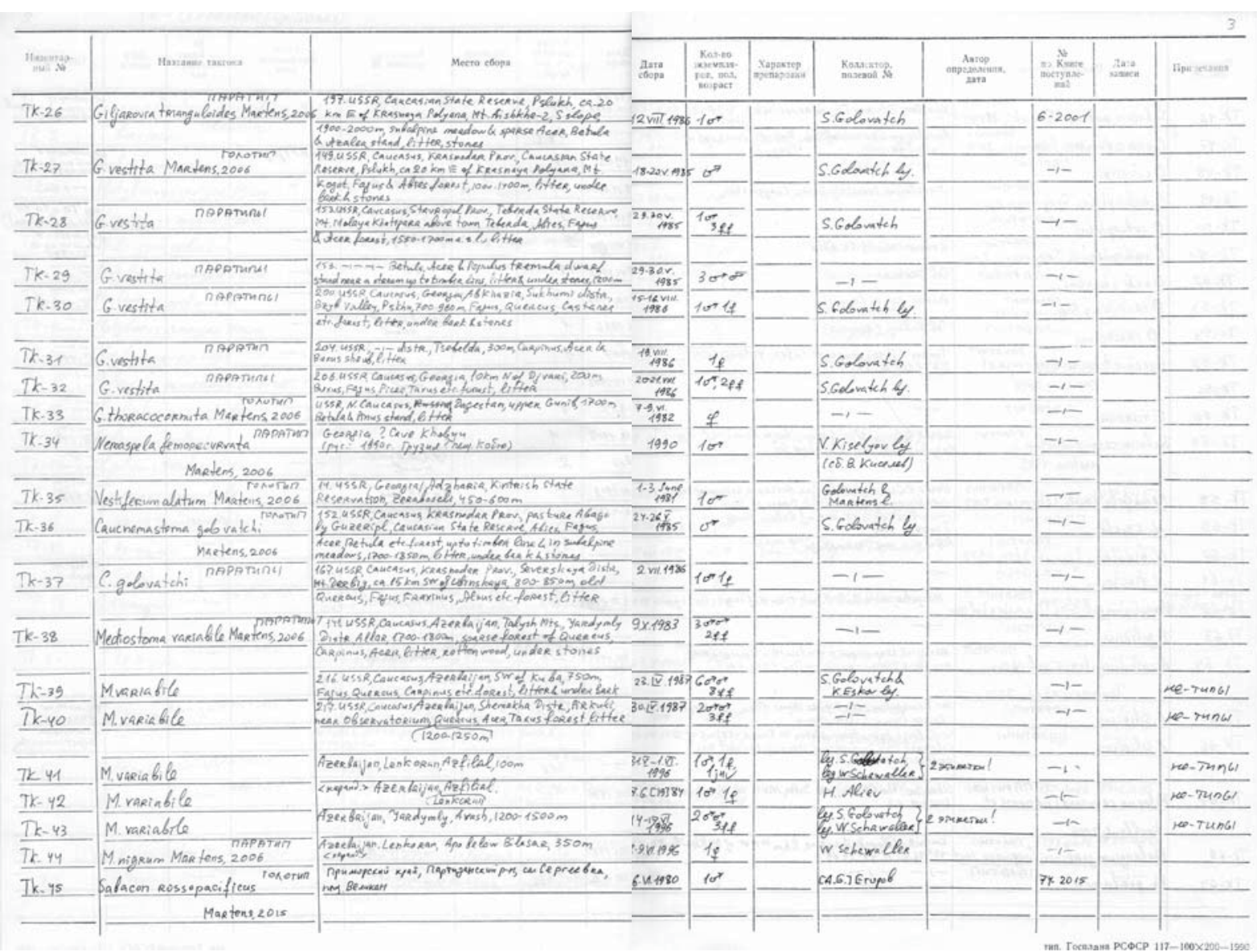

Fig. 2. The ZMMU inventory book of Opiliones (index Tk).

Рис. 2. Инвентарная книга сенокосцев (Opiliones, индекс Tk) Зоологического музея МГУ.

\section{List of the types}

alatum Martens, 2006, Vestiferum

Martens, 2006: 176-178, Abb. 18h-o, 20, 21c-d.

Tk-35. Holotype $\sigma^{7}, 14$. USSR, Georgia/Adzharia, Kintrish State Reservation, Zeraboseli, 450-600 m, 1-3 June 1981, Golovatch \& Martens 1.

The species was described from two specimens; a paratype male is kept in NHMW.

CURRENT STATUS. Vestiferum alatum Martens, 2006 (Nemastomatidae).

arborphilus Snegovaya, 2010, Opilio

Snegovaya, 2010: 7-9, figs 74-82.

Tk-49. Holotype O', Azerbaijan, Ismailly Distr., Chaygovshan, 23.07.2004, leg. N. Snegovaya.

Tk-50. Paratype: 1 , Azerbaijan, Ismailly Distr., Chaygovshan, 23.07.2004, leg. N. Snegovaya.

Other types are kept in IZBA and RCNS.

CURRENT STATUS. Opilio arborphilus Snegovaya, 2010 (Phalangiidae).

birsteini Ljovuschkin, 1972, Nemaspela

Ljovuschkin, 1972: 66-70, figs 2-3.

Tk-60. Holotype O', Абхазия, пещ. Таркиладзе $\{$ Abkhazia, Cave Tarkiladze\}, 7.VIII.1939, leg. Я.А. Бирштейн \{Ya.A. Birstein $\}$ [+ micropreparation of copulatory apparatus, a slide]
Tk-61. Paratype: 1 +, Абхазия, пещ. Таркиладзе $\{$ Abkhazia, Cave Tarkiladze\}, 7.VIII.1939, leg. Я.А. Бирштейн \{Ya.A. Birstein .

REMARK. No other paratypes mentioned in the original description are in ZMMU, likely being lost.

CURRENT STATUS. Nemaspela birsteini Ljovuschkin, 1972 (Nemastomatidae).

\section{birsteini Ljovuschkin, 1971, Taracus}

Ljovuschkin, 1971: 127-130, figs 1А-Д, 2А-Б, 3 (map).

Tk-1. Holotype $\sigma^{7}$, Приморский край, Партизанский район, пещера Белый Дворец \{Maritime Province, Partizansk Distr., Cave Belyi Dvorets\}, 15-20.IX.1966, собр. С.И. Левушкин \{leg. S.I. Ljovuschkin\}. [+micropreparation of copulatory apparatus, a slide]

Tk-2. Paratypes: 9 фо, Приморский край, Партизанский район, пещера Белый Дворец \{Maritime Province, Partizansk Distr., Cave Belyi Dvorets\}, 15-20.IX.1966, собр. С.И. Левушкин \{leg. S.I. Ljovuschkin\}.

Types are kept in ZMMU only.

CURRENT STATUS. Taracus birsteini Ljovuschkin, 1971 (Ischyropsalidae).

caucasicus Snegovaya, 2010, Opilio

Snegovaya, 2010: 9, figs 92-100.

Tk-53. Holotype $\sigma^{7}$, Russia, North Caucasus, 2003, leg. P. Kiyashko. 


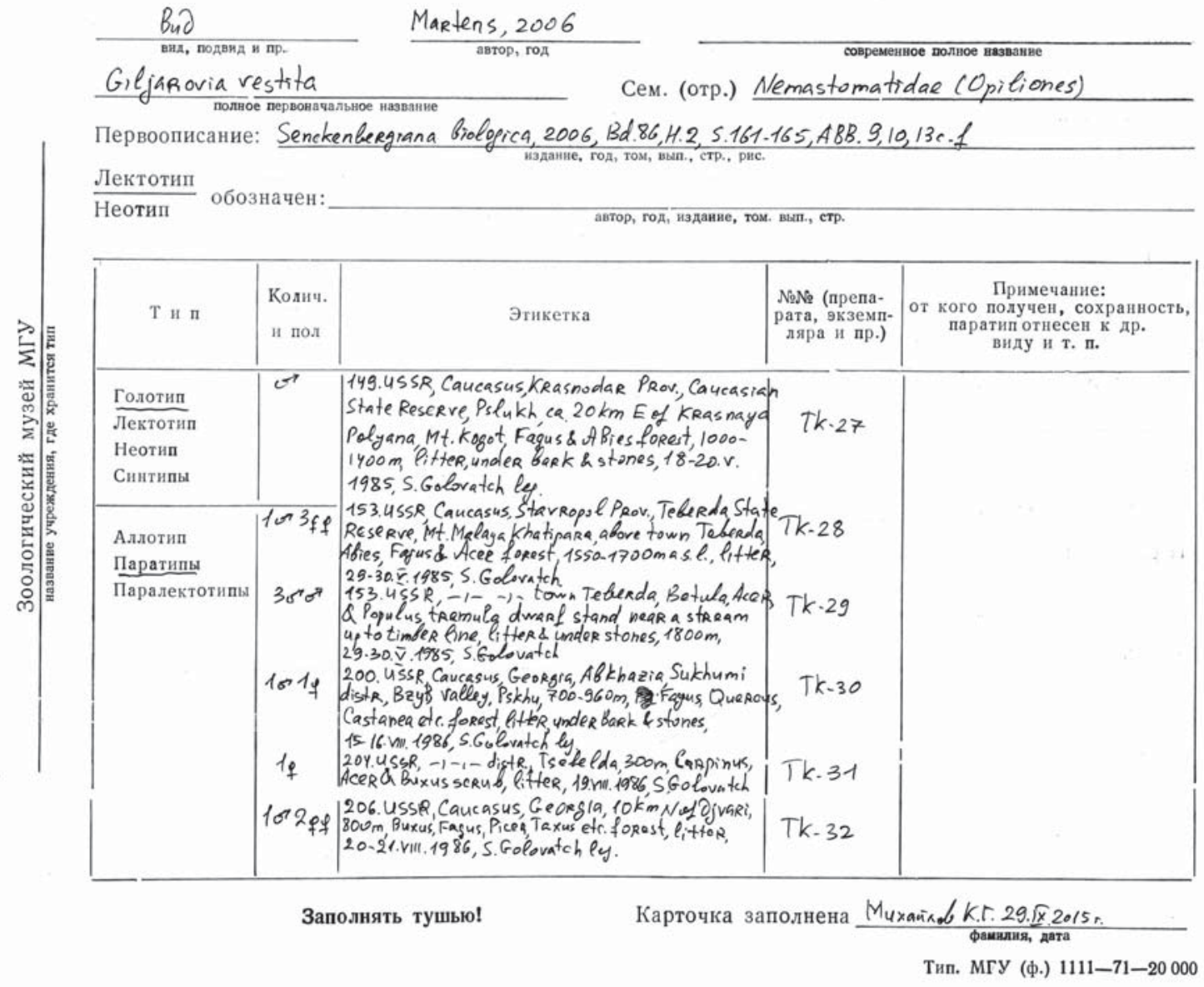

ПР И МЕ Ч А ИЯ

Тип переописан:

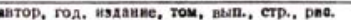

Вид сведен в синонимы:

Вид отнесен к другому роду:

Senokenberg Museum (Frankfurt am Marn, Germamy), pa Doval kourexusust Arbeitssammlury?

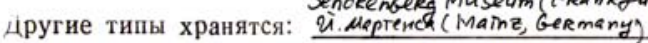

Паратипы посланы в обмен:

kyas, xorka

Тип исследовали:

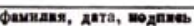

Fig. 3. An example of a ZMMU type card, recto and verso.

Рис. 3. Образец типовой карточки Зоологического музея МГУ, спереди и сзади. 


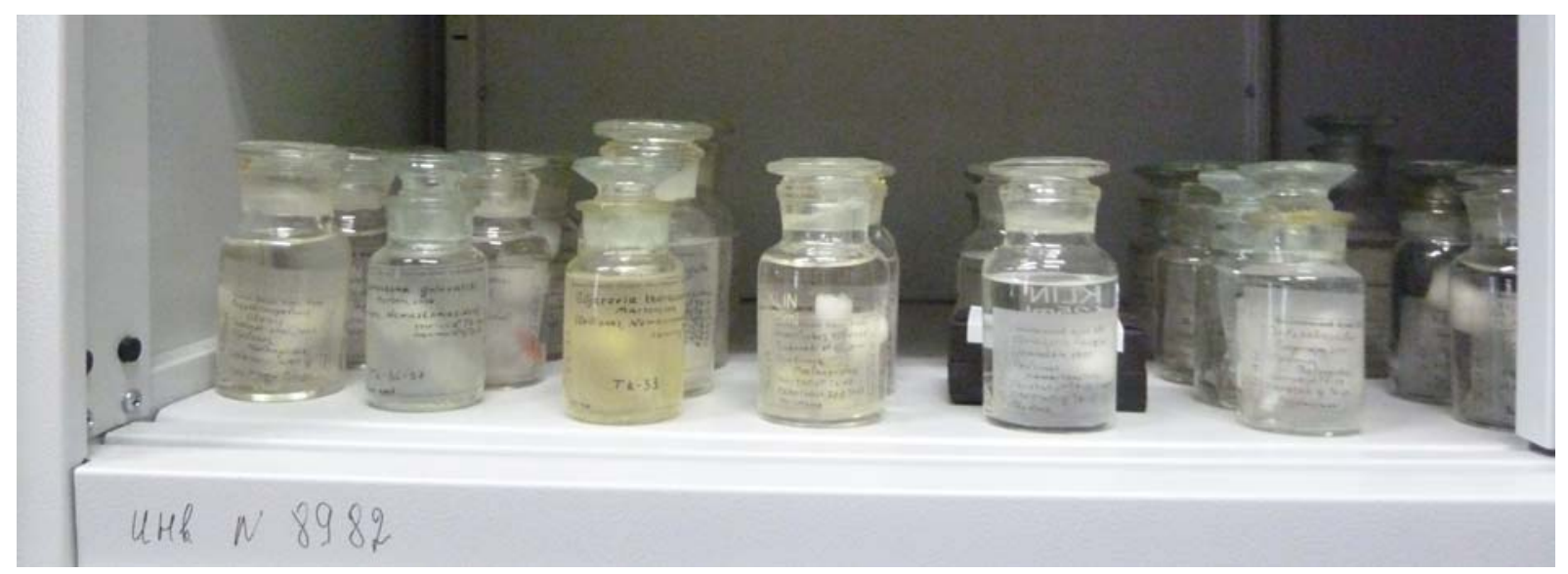

Fig. 4. The type collection of Opiliones in ZMMU.

Рис. 4. Типовая коллекция сенокосцев (Opiliones) Зоологического музея МГУ.

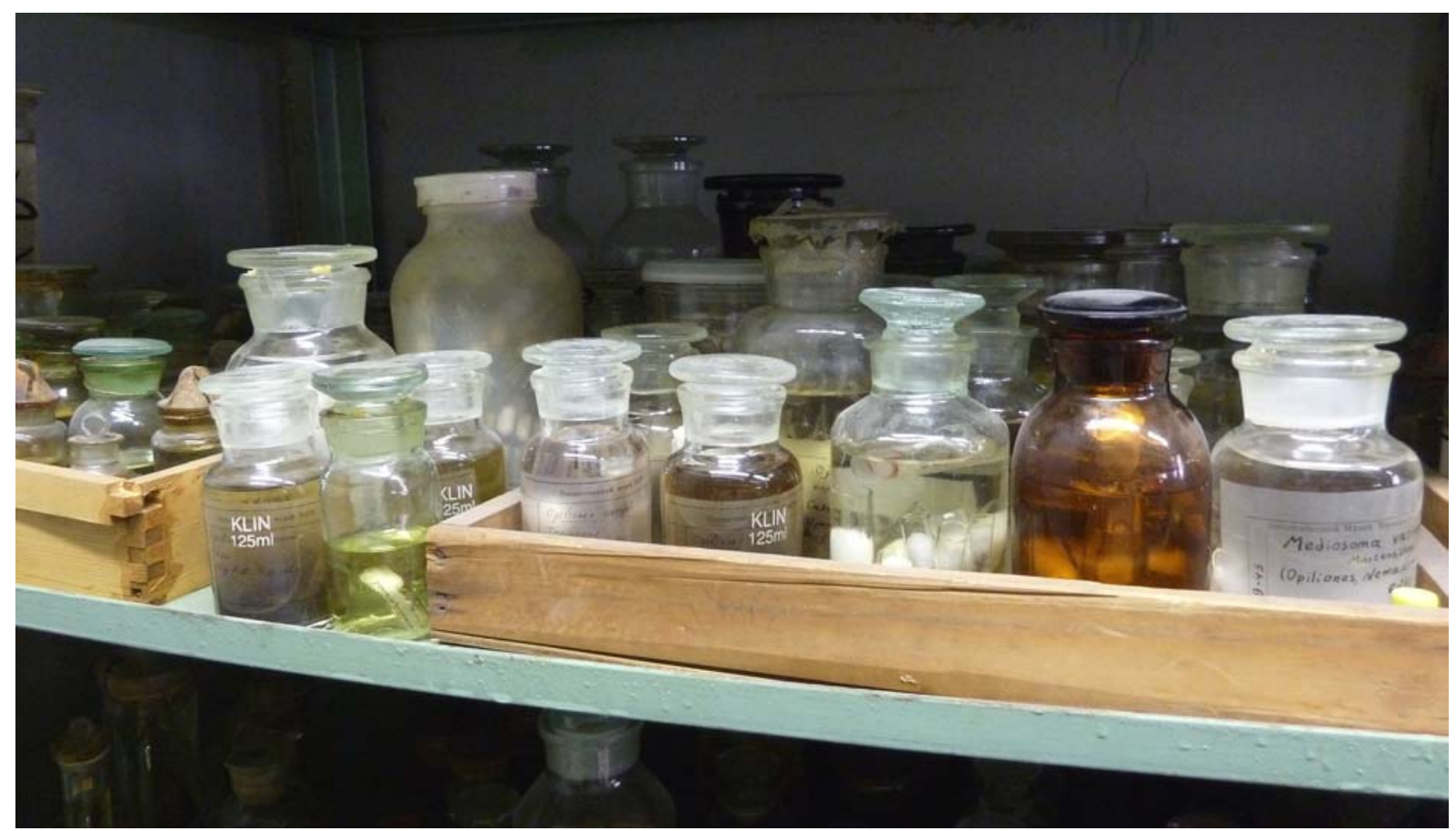

Fig. 5. The main collection of Opiliones in ZMMU.

Рис. 5. Основная коллекция сенокосцев (Opiliones) Зоологического музея МГУ.

Tk-54. Paratype: 1 , , Сев. Кавказ \{North Caucasus\}, <Лaгонаки \{Lagonaki\}>, <2003>, Кияшко П. \{Kiyashko P.\} Other types are kept in RCNS.

CURRENT STATUS. Opilio caucasicus Snegovaya, 2010 (Phalangiidae).

crimeana Chemeris $^{1}$ et Kovblyuk, 2006, Rilaena Chemeris, Kovblyuk, 2006: 318-322, figs 34-47, map 3.

Tk-67. Paratypes: $3 \sigma^{7} \sigma^{\top}, 3$ of , Ukraine: the Crimea, near Yalta, Nikita Vil., Mart'yan Cape Reserve, $\mathrm{p}<$ itfall $>$ $\mathrm{t}<$ raps $>$, 15.04.2000-4.06.2001, coll. M.M. Kovblyuk.

Other types are kept in ISEA (holotype) and TNU.

${ }^{1}$ In various papers, the name of A.N. Tchemeris is transliterated either as Tchemeris or Chemeris. The former option is preferable. In this list, both spellings are cited, according to the original papers.
CURRENT STATUS. Rilaena crimeana Chemeris et Kovblyuk, 2006 (Phalangiidae).

\section{femorecurvata Martens, 2006, Nemaspela}

Martens, 2006: 171-173, Abb. 14-15, 17.

Tk-34. Paratype: $1 \sigma^{7}$, Georgia, ? Cave Khobyu, 1990, V. Kiselyov leg. [Russian label:] 1990 г. Грузия, ? пещ. Кобю, сб. В. Киселев.

The species was described from two specimens; the holotype is kept in CJMM.

CURRENT STATUS. Nemaspela femorecurvata Martens, 2006 (Nemastomatidae).

gobiensis Tsurusaki, Tchemeris et Logunov, 2000,

\section{Homolophus}

Tsurusaki et al., 2000: 80-83, figs 6-8. 
Tk-62. Holotype $\sigma^{7}$, Mongolia, South Gobi Aimak, 15 km W of Tsogt-Tsetsy, 1.09.1986, coll. A.P. Rasnitsyn.

Tk-63. Paratypes: 2 우, Mongolia, South Gobi Aimak, $15 \mathrm{~km} \mathrm{~W}$ of Tsogt-Tsetsy, 1.09.1986, coll. A.P. Rasnitsyn. Types are kept in ZMMU only.

REMARK. On the original label Tk-62, the name of the collector was erroneously given as A.N. Rasnitsyn, instead of A.P. Rasnitsyn.

CURRENT STATUS. Homolophus gobiensis Tsurusaki, Tchemeris et Logunov, 2000 (Phalangiidae).

golovatchi Martens, 2006, Caucnemastoma

Martens, 2006: 180-182, Abb. 17, 19, 21i-k.

Tk-36. Holotype $\sigma^{\top}, 152$. USSR, Caucasus, Krasnodar Prov., pasture Abago by Guzeripl, Caucasian State Reserve, Abies, Fagus, Acer, Betula etc. forest, up to tinber line \&in subalpine meadows, $1700-1850 \mathrm{~m}$, litter, under bark \& stones, 24-26.V.1985, S. Golovatch leg.

Tk-37. Paratypes: $1 \sigma^{7}, 1$ ㅇ, 167. USSR, Caucasus, Krasnodar Prov., Mt. Derbiy, ca. 15 km SW of Ubinskaya, 800-850 m, old Quercus, Fagus, Fraxinus, Alnus etc. forest, litter, 2.VII.1986, S. Golovatch leg.

Other types are kept in CJMM and SMF.

CURRENT STATUS. Caucnemastoma golovatchi Martens, 2006 (Nemastomatidae).

\section{gorbunovi Snegovaya, 2014, Phalangium}

Snegovaya, 2014: 6-7, figs 113-127, 129 (map).

Tk-68. Holotype $\sigma^{7}$, Russia, Volgograd region, $2 \mathrm{~km}$ WNW of Mikhailovka, 49 $46.80^{\prime} \mathrm{N}, 44^{\circ} 24.09^{\prime} \mathrm{E}, 15$ 17.05.2003, leg. O. Gorbunov.

Tk-69. Paratype: 1 , Russia, Volgograd region, $2 \mathrm{~km}$ WNW of Mikhailovka, $49^{\circ} 46.80^{\prime} \mathrm{N}, 44^{\circ} 24.09^{\prime} \mathrm{E}, 15$ 17.05.2003, leg. O. Gorbunov.

Other types are kept in RCNS.

CURRENT STATUS. Phalangium gorbunovi Snegovaya, 2014 (Phalangiidae).

hirsutus Snegovaya in Snegovaya et Chumachenko, 2011, Calathocratus

Snegovaya, Chumachenko, 2011: 117, figs 2-14.

Tk-70. Holotype $\sigma^{7}$, Russia, Sochi, yew and box-tree grove, yew-beech (area 1), October 2006, soil trap № 4, leg. Yu. Chumachenko.

Tk-71. Paratype: 1 , , Russia, Sochi, yew and box-tree grove, yew-beech (area 1), October 2006, soil trap № 4, leg. Yu. Chumachenko.

Other types are kept in RCNS, SMF, TTU-Z, and ZIN.

CURRENT STATUS. Calathocratus hirsutus Snegovaya in Snegovaya et Chumachenko, 2011 (Trogulidae).

kovali Chemeris, 2009, Nemaspela map 1 .

Chemeris, 2009: 293-294, figs 7-8, 17-18, 21, 26, 35-39,

Tk-58. Paratype: 1 ㅇ, Russia, C. Caucasus, KabardinoBalkaria, canyon of the upper stream of River Nal'chik, Omega-15 Cave, 28.05.1998, coll. A.G. Koval'.

Tk-59. Paratype: $1 \sigma^{7}$, Russia, C. Caucasus, KabardinoBalkaria, source of Belaya River, Fontanka Cave, 21.05.1998, A.G. Koval'.

Other types are kept in ISEA and ZIN (holotype).

CURRENT STATUS. Nemaspela kovali Chemeris, 2009

(Nemastomatidae).

minutus Snegovaya in Snegovaya et Chumachenko, 2011, Calathocratus

Snegovaya, Chumachenko, 2011: 117, figs 15-27.
Tk-72. Holotype $\sigma^{7}$, Russia, Sochi, yew and box-tree grove, beech forest, May 2006, soil trap № 6, leg. Yu. Chumachenko.

Tk-73. Paratype: $1+$, Russia, Sochi, yew and box-tree grove, beech forest, May 2006, soil trap № 6, leg. Yu. Chumachenko.

Other types are kept in RCNS, SMF, TTU-Z, and ZIN.

CURRENT STATUS. Calathocratus minutus Snegovaya in Snegovaya et Chumachenko, 2011 (Trogulidae).

nabozhenkoi Snegovaya, 2010, Opilio

Snegovaya, 2010: 9, figs 83-91.

Tk-51. Holotype $\sigma^{7}$, Russia: North Ossetia, 25.07.2006, leg. M. Nabozhenko.

Tk-52. Paratype: 1 + Сев. Осетия \{North Ossetia\}, 25.07.2006, Набоженко А.B. \{Nabozhenko A.V.\}

Other types are kept in IZBA, RCNS, and ZIN.

CURRENT STATUS. Opilio nabozhenkoi Snegovaya, 2010 (Phalangiidae).

\section{nigrum Martens, 2006, Mediostoma}

Martens, 2006: 190-192, Abb. 20, 23o-q, 24a-e, 25a-b.

Tk-44. Paratype: $1+$, [in pencil] Azerbaijan, Lenkoran, Apo below Bilasar, 350 m, 8-9.VI.1996, W. Schawaller. type).

Other types are kept in CJMM, NHNW, and SMF (holo-

CURRENT STATUS. Mediostoma nigrum Martens, 2006 (Nemastomatidae).

rossopacificus Martens, 2015, Sabacon

Martens, 2015: 204-206, figs 220-228.

Tk-45. Holotype $\sigma^{\top}$, Приморский край, Партизанский p-н, сел. Сергеевка, пещера Великан \{Maritime Province, Partizansk Distr., Sergeevka, Cave Velikan\}, 6.VI.1980, $<$ А.Б. $\{$ А.В. $\}>$ Егоров $\{$ Egorov $\}$.

One more paratype is kept in SMF.

CURRENT STATUS. Sabacon rossopacificus Martens, 2015 (Sabaconidae).

sergeidedicatum Martens, 1989, Sabacon

Martens, 1989: 372-376, Abb. 7-11, 13, 15-16.

Tk-57. Holotype $\sigma^{7}$, UdSSR, Sibirien, Altai-Gebirge, Cherga, beim Dorf Artybash, nahe dem Teletzkoye See, 713.7.1982, S.I. Golovatch leg.

One more paratype (male) is kept in SMF.

CURRENT STATUS. Sabacon sergeidedicatum Martens, 1989 (Sabaconidae).

sibiricus Tsurusaki, Tchemeris et Logunov, 2000, Acanthomegabunus

Tsurusaki et al., 2000: 74-79, figs 1-4.

Tk-64. Paratype: 1 ○', Хакасия, Ширинский p-н, $\sim 1$ км Ю п. Коммунар, мохово-каменистая лесотундра, \{Khakasia, Shira Distr., ca $1 \mathrm{~km} \mathrm{~S}$ of Kommunar, moss-stony forested tundra $\}$ 1300-1400 м $\{\mathrm{m}\}, 23.7 .1990$, сб. Д.В. Логунов \{leg. D.V. Logunov\}.

Tk-65. Paratypes: $2 \sigma^{7} \sigma^{7}$, юг Кемеровской области, Горная Шория, 10 км сев п. Шерегеш, г. Пустаг, гольцы, \{south of Kemerovo Area, Montane Shoria, $10 \mathrm{~km} \mathrm{~N}$ of Sheregesh, Mt Pustag, goltsy, =stony screes $\} \mathrm{h}=1300-1500$ м $\{m\}, 13-26.06 .1999$, D.E. Lomakin leg.

Tk-66. Paratypes: $1 \sigma^{7}, 1$ +, $\mathrm{W}-$ Siberia, KuznetskiyAlatau Mt. Range, $\sim 50 \mathrm{~km} \mathrm{~S}$ of Belogorsk Town, Tsemodan and Stanovoy Khrebet Mts, anemone meadow, 23.07.1992, N.B. Demidenko. 
Other types are kept in ISEA (holotype) and the Department of Biology, Faculty of Education, Tottori University (Japan).

REMARK. The correct name of the mountain in Tk-66 is Tshemodan, not Tsemodan.

CURRENT STATUS. Acanthomegabunus sibiricus Tsurusaki, Tchemeris et Logunov, 2000 (Phalangiidae).

silvestris Snegovaya, 2010, Opilio

Snegovaya, 2010: 7, figs 65-73.

Tk-47. Holotype $\sigma^{7}$, Azerbaijan: Shemakha Distr., Pirgulu, environs of Sis village, 19-22.05.2004, leg. N. Snegovaya, Kh. Aliyev.

Tk-48. Paratype: 1 , Azerbaijan: Shemakha Distr., Pirgulu, environs of Sis village, 19-22.05.2004, leg. N. Snegovaya, Kh. Aliyev.

Other types are kept in IZBA, RCNS, and ZIN.

CURRENT STATUS. Opilio silvestris Snegovaya, 2010 (Phalangiidae).

\section{thoracocornuta Martens, 2006, Giljarovia}

Martens, 2006: 167-169, Abb. 12g-h, 13g-h, 20.

Tk-33. Holotype + , USSR, N Caucasus, Dagestan, upper Gunib, 1700 m, Betula \& Pinus stand, litter, 7-9.VI.1982, S. Golovatch leg.

The species was described from the holotype alone.

CURRENT STATUS. Giljarovia thoracocornuta Martens, 2006 (Nemastomatidae).

\section{triangula Martens, 2006, Giljarovia}

Martens, 2006: 156-159, Abb. 4-5, 8, 13i-k

Tk-16. Holotype $\sigma^{7}, 77$. USSR, Caucasus Major, Georgia, Kazbegi, $2000 \mathrm{~m}$, in forests of Betula \& Pinus, in meadows, litter \& under stones, 2-6.VI.1982, <S.I.> Golovatch leg.

Tk-17. Paratypes: 9 우, 77. USSR, Caucasus Major, Georgia, Kazbegi, $2000 \mathrm{~m}$, in forests of Betula \& Pinus, in meadows, litter \& under stones, 2-6.VI.1982, <S.I.> Golovatch leg.

Tk-19. Paratypes: $10^{\top}, 3$ o+, 153. USSR, Caucasus, Stavropol Prov., Teberda State Reserve, Mt. Malaya Khatipara above Town Teberda, Betula, Acer \& Populus tremula dwarf stand near a stream up to timber line, litter \& under stones, 1800 m, 29-30.V.1985, S. Golovatch.

Tk-20. Paratypes: $1 \overbrace{}^{7}, 2$ 우, 155. USSR, Caucasus, Stavropol Prov., Teberda State Reserve, Canyon Gonachkhir, between Teberda and Dombai, road to Klukhor Pass, 1700-1800 m, Abies, Fagus, Acer..., <litter, under bark \& stones, $>$ 1.VI.1985, S. Golovatch leg.

Tk-21. Paratypes: $2 \bigcirc^{7} \sigma^{7}, 4$ $9+, 159$. USSR, Caucasus, Stavropol Prov., Teberda State Reserve, Kizgich Canyon, N of Arkhyz, Abies, Picea, Pinus, Fagus, Betula \& Acer forest, litter, under bark \& stones, 1550-1650 m, 5.VI.1985, S. Golovatch leg.

Tk-22. Paratypes: $1 O^{\top}, 3$ 운, 159. USSR, Caucasus, Stavropol Prov., Teberda State Reserve, Kizgich Canyon, N of Arkhyz, riverine Alnus \& Betula wet forests, 1450-1500 m, 5.VI.1985, S. Golovatch leg.

Tk-23. Paratype: $1 \sigma^{7}, 178$. USSR, Caucasus, Kabardino-Balkaria, Chegem Distr., $5 \mathrm{~km} \mathrm{~S}$ of Upper Chegem, timberline of Betula, Rhododendron \& Juniperus, litter, under stones, 2100-2200 m, 13.VII.1986, leg. S. Golovatch.

Other types are kept in CJMM and SMF.

REMARK. Possibly the habitat label in Tk-21 is incorrect, see under Tk-22.
CURRENT STATUS. Giljarovia triangula Martens, 2006 (Nemastomatidae).

trianguloides Martens, 2006, Giljarovia

Martens, 2006: 159-161, Abb. 6-8.

Tk-24. Holotype $\sigma^{7}, 149$. USSR, Caucasus, Krasnodar Prov., Caucasian State Reserve, Pslukh, ca. $20 \mathrm{~km}$ E of Krasnaya Polyana, 18-20.V.1985, S. Golovatch leg.

Tk-25. Paratypes: 1 フ , 8 우, 199. USSR, Caucasian State Reserve, Krasnaya Polyana, 600-750 m, Quercus, Fagus, Castanea, Carpinus etc. forest, litter, bark, stones, 8 9.VIII.1986, <S.I. $>$ Golovatch.

Tk-26. Paratype: $1{ }^{7}, 197$. USSR, Caucasian State Reserve, Pslukh, ca. $20 \mathrm{~km}$ E of Krasnaya Polyana, Mt. Aishkho-2, S slope, 1900-2000 m, subalpine meadow \& sparse Acer, Betula \& Azalea stand, litter, stones, 12.VIII.1986, S. Golovatch.

Other types are kept in CJMM and SMF.

CURRENT STATUS. Giljarovia trianguloides Martens, 2006 (Nemastomatidae).

turcicus Snegovaya ey Marusik, 2012, Egaenus

Snegovaya, Marusik, 2012: 68, figs 44-53.

Tk-55. Holotype $\sigma^{2}$, Turkey, T-10, Bursa, Nilüfer, $40^{\circ}$ $07.466^{\prime} \mathrm{N}, 28^{\circ} 42.105^{\prime} \mathrm{E}, 570 \mathrm{~m}, 2.06 .2009$, Yu.M. Marusik.

Tk-56. Paratype: 1 . Turkey, T-10, Bursa, Nilüfer, $40^{\circ}$ $07.466^{\prime} \mathrm{N}, 28^{\circ} 42.105^{\prime} \mathrm{E}, 570 \mathrm{~m}, 2.06 .2009$, Yu.M. Marusik. One more paratype $\left(\mathrm{O}^{7}\right)$ is kept in RCNS.

CURRENT STATUS. Egaenus turcicus Snegovaya et Marusik, 2012 (Phalangiidae).

variabile Martens, 2006, Mediostoma

Martens, 2006: 185-189, Abb. 20, 23a-h, 24k-q, 25i-k.

Tk-38. Paratypes: $3 \sigma^{7} \sigma^{7}, 2$ 우, 141. USSR, Caucasus, Azerbaijan, Talysh Mts., Yardymly Distr., Allar, 1700-1800 $\mathrm{m}$, sparse forest of Quercus, Carpinus, Acer, litter, rotten wood, under stones, 9.X.1983, S. Golovatch leg.

Other types are kept in CJMM and SMF (holotype).

REMARK. In addition, the specimens listed as nontypes in the original description are kept in ZMMU under numbers Tk-39-43.

CURRENT STATUS. Mediostoma variabile Martens, 2006 (Nemastomatidae)

\section{vestita Martens, 2006, Giljarovia}

Martens, 2006: 161-165, Abb. 9, 10, 13c-f.

Tk-27. Holotype $\sigma^{7}, 149$. USSR, Caucasus, Krasnodar Prov., Caucasian State Reserve, Pslukh, ca. $20 \mathrm{~km} \mathrm{E}$ of Krasnaya Polyana, Mt. Kogot, Fagus \& Abies forest, 1000$1400 \mathrm{~m}$, litter, under bark \& stones, 18-20.V.1985, S. Golovatch leg.

Tk-28. Paratypes: 1 ○', 3 우, 153. USSR, Caucasus, Stavropol Prov., Teberda State Reserve, Mt. Malaya Khatipara above Town Teberda, Abies, Fagus, \& Acer forest, 1550-1700 m a.s.1., litter, 29-30.V.1985, S. Golovatch.

Tk-29. Paratypes: $3 \sigma^{7} \sigma^{7}, 153$. USSR, Caucasus, Stavropol Prov., Teberda State Reserve, Mt. Malaya Khatipara above Town Teberda, Betula, Acer \& Populus tremula dwarf stand near a stream up to timber line, litter \& under stones, 1800 m, 29-30.V.1985, S. Golovatch.

Tk-30. Paratypes: $1 \sigma^{7}, 1$ क , 200. USSR, Caucasus, Georgia, Abkhazia, Sukhumi distr., Bzyb Valley, Pskhu, 700-960 m, Fagus, Quercus, Castanea etc. forest, litter, under bark \& stones, 15-16.VIII.1986, S. Golovatch leg. 
Tk-31. Paratype: 1 ㅇ, 204. USSR, Caucasus, Georgia, Abkhazia, Sukhumi distr., Tsebelda, 300 m, Carpinus, Acer \& Buxus scrub, litter, 19.VIII.1986, S. Golovatch.

Tk-32. Paratypes: $1 \overbrace{}^{\top}, 2$ o+ , 206. USSR, Caucasus, Georgia, 10 km N of Djvari, 800 m, Buxus, Fagus, Picea, Taxus etc. forest, litter, 20-21.VIII.1986, S. Golovatch leg. Other types are kept in CJMM and SMF.

CURRENT STATUS. Giljarovia vestita Martens, 2006 (Nemastomatidae).

Acknowledgements. I am cordially obliged to my colleague and friend Sergei I. Golovatch (Moscow, Russia) for strongly enriching the ZMMU arachnid collection. He also rendered linguistic help. Dmitry V. Logunov (Manchester, Great Britain) and Alexander V. Sysoev (ZMMU) kindly reviewed this paper and made very useful comments. This paper is supported by Russian Science Foundation Project No. 14-5000029. The inventory book-keeping is supported by Moscow State University Project No. AAAA-A16116021660077-3.

\section{References}

Borutzky E.V. 1972. [A list of the holotypes of Isopoda Oniscoidea of the Zoological Museum of Moscow University] // Archives (Sbornik trudov) of the Zoological Museum of Moscow State University. Vol.12. P.191-200 [in Russian].

Chemeris A.N. 2009. New data on the harvestman genus Nemaspela Silhavý, 1966 (Arachnida: Opiliones) // Bull. Brit. arachnol. Soc. Vol.14. Pt.7. P.286-296.

Chemeris A.N., Kovblyuk M.M. 2006. A contribution to the knowledge of the harvestman fauna of the Crimea (Arachnida: Opiliones) // Arthropoda Selecta. Vol.14 (for 2005). No.4. P.305328

Ivanov D.L., Sysoev A.V. 2000. Types of Mollusca in the Zoological Museum of Moscow University // Archives of the Zoological Museum of Moscow State University. Vol.40. 187 p.

Ljovuschkin S.I. 1971. [Biospeologica Sovietica XLIII. Report on the first record of the American genus Taracus Simon, 1879 (Opiliones, Ischyropsalididae [sic!]) in Asia, with description of $T$. birsteini sp. n. from a cave in South Primorie] // Bulletin of the Moscow Society of Naturalists. Sect. Biol. Vol.76. No.3. P.127-133 [in Russian].

Ljovuschkin S.I. 1972. [Harvestmen of the family Nemastomatidae from caves of USSR] // Archives (Sbornik trudov) of Zoological Museum, Moscow State University. Vol.12. P.61-73 [in Russian].
Martens J. 1989. Sibirische Arten der Gattung Sabacon Simon 1879 (Arachnida: Opiliones: Sabaconidae) // Senckenbergiana biol. Bd.69. H.4/6. S.369-377.

Martens J. 2006. Weberknechte aus dem Kaukasus (Arachnida, Opiliones, Nemastomatidae) // Senckenbergiana biologica. Bd.86. H.2. S.145-210.

Martens J. 2015. Sabacon Simon, 1879 in the Palaerctic: A survey of new and known species from France, Nepal, India, China, Russia and Japan (Arachnida: Opiliones: Sabaconidae) // M. Hartmann, J. Weipert (Hrsg.). Biodiversität und Naturausstattung im Himalaya. Erfurt: Naturkundemuseum. Bd.5. P.167210.

Mikhailov K.G. 2016. [Arachnology in Russia/USSR] // Pavlinov I.Ya., Kalyakin M.V., Sysoev A.V. (eds.). Aspects of biodiversity. Part 2. Archives of Zoological Museum of Moscow State University. Vol.54. No.2. P.655-691 [in Russian, with English summary].

Pakhnevich A.V., Zezina O.N., Temereva E.N. 2013. [Recent brachiopods and phoronids in the collection of Zoological Museum of the Moscow State University] // Zoologicheskie Issledovania. Moscow: KMK Sci. Press. No.13. 48 p. [in Russian, with English summary].

Perel' T.S., Sokolskaya N.L. 1972 [Types of Oligochaeta in the collection of Zoological Museum MGU] // Archives (Sbornik trudov) of the Zoological Museum of Moscow State University. Vol.12. P.201-204 [in Russian].

Snegovaya N.Yu. 2010. Further studies on harvestmen of the genus Opilio Herbst, 1798 (Arachnida: Opiliones: Phalangiidae) from the Caucasus // Caucasian Entomol. Bull. Vol.6. No.1. P.3-18.

Snegovaya N.Yu. 2014. Survey of the genus Phalangium Linnaeus, 1758 (Phalangiidae: Opiliones) from the Caucasus with description of two new species // Fragmenta Faunistica. Vol.57. No.1. P.1-19.

Snegovaya N.Yu., Chumachenko Yu.A. 2011. Harvestmen (Arachnida: Opiliones) from the yew and box-tree grove of the Caucasian State Natural Biospheric Reserve, Russia // Caucasian Entomol. Bull. Vol.7. No.2. P.115-124.

Snegovaya N.Yu., Marusik Yu.M. 2012. New species and collections of Opiliones (Arachnida) from Turkey // Acta Arachnologica. Vol.61. No.2. P.59-70.

Tsurusaki N., Tchemeris A.N., Logunov D.V. 2000. Two new species of Opiliones from southern Siberia and Mongolia, with an establishment of a new genus and redefinition of the genus Homolophus (Arachnida: Opiliones: Phalangiidae) // Acta Arachnologica. Vol.49. No.1. P.73-86.

Vinogradov G.M., Vinogradov M.E. 2006. [Type specimens of pelagic amphipods (Crustacea: Amphipoda) described by J.A. Birstein and M.E. Vinogradov, deposited in the Zoological Museum of Moscow State University] // Invertebrate Zoology. Vol.3. No.1. P.53-64 [in Russian, with English summary].

Responsible editor S.I. Golovatch 